\title{
Physical Organohydrogels With Extreme Strength and Temperature Tolerance
}

\begin{abstract}
Jing Wen Zhang 1,2†, Dian Dian Dong 1,2†, Xiao Yu Guan 1,2, En Mian Zhang ${ }^{3}$, Yong Mei Chen ${ }^{1,2,3 *}$, Kuan Yang ${ }^{1,2}$, Yun Xia Zhang ${ }^{4}$, Malik Muhammad Bilal Khan ${ }^{1,2}$, Yasir Arfat ${ }^{1,2}$ and Yasir Aziz ${ }^{1,2}$

${ }^{1}$ College of Bioresources Chemical and Materials Engineering, Shaanxi University of Science \& Technology, Xi'an, China, ${ }^{2}$ National Demonstration Center for Experimental Light Chemistry Engineering Education (Shaanxi University of Science \& Technology), Xi'an, China, ${ }^{3}$ State Key Laboratory for Strength and Vibration of Mechanical Structures, International Center for Applied Mechanics, School of Aerospace Engineering, Xi'an Jiaotong University, Xi'an, China, ${ }^{4}$ Research Center for Semiconductor Materials and Devices, College of Arts and Sciences, Shaanxi University of Science \& Technology, Xi'an, China
\end{abstract}

Tough gel with extreme temperature tolerance is a class of soft materials having potential applications in the specific fields that require excellent integrated properties under subzero temperature. Herein, physically crosslinked Europium (Eu)-alginate/polyvinyl alcohol (PVA) organohydrogels that do not freeze at far below $0^{\circ} \mathrm{C}$, while retention of high stress and stretchability is demonstrated. These organohydrogels are synthesized through displacement of water swollen in polymer networks of hydrogel to cryoprotectants (e.g., ethylene glycol, glycerol, and d-sorbitol). The organohydrogels swollen water-cryoprotectant binary systems can be recovered to their original shapes when be bent, folded and even twisted after being cooled down to a temperature as low as -20 and $-45^{\circ} \mathrm{C}$, due to lower vapor pressure and ice-inhibition of cryoprotectants. The physical organohydrogels exhibit the maximum stress (5.62 $\pm 0.41 \mathrm{MPa})$ and strain $(7.63 \pm 0.02)$, which is about 10 and 2 times of their original hydrogel, due to the synergistic effect of multiple hydrogen bonds, coordination bonds and dense polymer networks. Based on these features, such physically crosslinked organohydrogels with extreme toughness and wide temperature tolerance is a promising soft material expanding the applications of gels in more specific and harsh conditions.

Keywords: organohydrogels, high strength, anti-freezing, non-drying, temperature tolerance

\section{INTRODUCTION}

Hydrogels are the typical soft materials, by virtue of their great potentials in applications spanning from soft robotics, sensors, actuators to tissue engineering (Wegst et al., 2014; Iwaso et al., 2016; Kim et al., 2016; Banerjee et al., 2018; Dong et al., 2018; Hu et al., 2019). Nevertheless, conventional hydrogels are considered to be mechanically weak due to lack of an effective energy dissipation mechanism or intrinsic structural heterogeneity (Dhivya et al., 2015; Yuk et al., 2016), limiting utilization in some fields that require excellent mechanical properties (Gao et al., 2016; Fan et al., 2019; Lai et al., 2019). Therefore, improving mechanical properties of hydrogels became an important research hotspot. So far, versatile strategies to achieve tough hydrogels have been emerged, including double-network hydrogels (Gong et al., 2003; Gong, 2014; Liang et al., 2016; Chen et al., 2018; Jing et al., 2019), nanocomposite hydrogels (Haraguchi and Takehisa, 2002; Chen et al., 2015; GhavamiNejad et al., 2016; Liu Y. et al., 2017; Zhu et al., 2017), topological 
hydrogels (Okumura and Ito, 2001; Li et al., 2018), macromolecular microsphere composite hydrogels (Huang et al., 2007; Gu et al., 2016; Zhang and Khademhosseini, 2017; Wang Z. et al., 2018), hydrophobic association hydrogels (Li et al., 2012; Mihajlovic et al., 2017; Han et al., 2018), hydrogen bonding/dipole-dipole reinforced hydrogels (Han et al., 2012; Zhang et al., 2015; Qin et al., 2018), and many others (Gong et al., 2016; Liu J. et al., 2017; Zhao et al., 2019). However, almost all of the hydrogels swollen a large amount of water in polymer networks cannot resist a cold or hot environment (Wei et al., 2014, 2015; Wang W. et al., 2018), hindering the application of tough hydrogels in harsh conditions. Subzero temperature results in freezing of hydrogels, while high temperature lead to drying (Rong et al., 2017; Zhang et al., 2018). Freezing and drying cause the hydrogels to hard, opaque and dry, which undoubtedly change the integrated mechanical properties of hydrogels, leading to unstable nature under wide temperature range (Han et al., 2017; Lou et al., 2019). So far, it is still a challenge to design a hydrogel with enhanced and tunable mechanical strength together with extreme temperature tolerance.

Recently, two approaches have been proposed to develop hydrogels with extreme temperature tolerance. One is introduction of an ionic compound (e.g., $\mathrm{NaCl}, \mathrm{LiCl}$, and $\mathrm{CaCl}_{2}$ ) to hydrogels, i.e., the polymer networks swollen with salt water (Morelle et al., 2018), for dropping the ice point of water according to the principle of colligative properties of solution. However, water can be evaporated from polymer networks under high temperature causing unstable mechanical properties. The other strategy is the introduction of a water-cryoprotectant binary solvent system into organohydrogel (OHG) networks through synthesis or displacement (Gao et al., 2017; Rong et al., 2018). Compared with the hydrogel containing ionic compound, water-cryoprotectant binary solvent endows stable mechanical properties to gels under both low and high temperature, due to the advantages of cryoprotectants including relatively high volatile point and inhibition ice crystallization (Elliott et al., 2017). The cryoprotectants, including ethylene glycol (EG), glycerol (GC), and d-sorbitol (SB) are suitable choices for fabricating organhydrogels swollen water-cryoprotectant binary solvents, which was firstly reported by Wang's group (Shi et al., 2017).

In the present study, we found that the mechanical properties and temperature tolerance could be dramatically enhanced by fabricating tough physically crosslinked organhydrogels via solvent displacement approach. The tough organohydrogels were prepared through displacing cryoprotectants (i.e., EG, GC, SB) into our previously reported Eu-alginate/PVA hydrogel networks mainly crosslinked by hydrogen bonds formed among PVA polymers and coordination bonds between $\mathrm{Na}$-alginate networks and $\mathrm{Eu}^{3+}$ ions (Wang et al., 2015; Hu et al., 2017). Multiple hydrogen bonds forming among cryoprotectants and PVA polymers enhance mechanical properties of organohydrogels. Moreover, cryoprotectants disrupt the formation of ice crystal lattices of the residual water, endowing extreme toughness and temperature tolerance to the organohydrogels. Furthermore, tunable mechanical performance of the oganohydrogels can be controlled by either selecting cryoprotectants or by varying the extent of solvent displacement. Therefore, physically crosslinked organohydrogels with enhanced and tunable mechanical properties, as well as extreme temperature tolerance could be designed and synthesized, potentially expanding scientific research and practical applications of gels.

\section{EXPERIMENT}

\section{Materials and Methods}

Polyvinyl alcohol (PVA, $\mathrm{Mn}=205,000)$ and sodium alginate $(\mathrm{Na}-$ alginate) were purchased from Sigma-Aldrich (Shanghai, China). Alginate is a linear copolymer of $\alpha$-L-guluronic acid ( $\mathrm{G}$ unit) and $\beta$-D-mannuronic acid ( $M$ unit). Europium chloride hexahydrate $\left(\mathrm{EuCl}_{3} \cdot 6 \mathrm{H}_{2} \mathrm{O}\right)$ was obtained from Qufu Chemical Co. Ltd. (Qufu China). Ethylene glycol, glycerol, and d-sorbitol were supplied by Cheng Jie Chemical Engineering Co. Ltd. (Shanghai, China). All chemicals were received and used without further purification. Ultrapure water with a resistivity higher than $18.2 \mathrm{M} \Omega \cdot \mathrm{cm}$ was supplied by a Millipore Simplicity 185 system, which was deoxygenated three times by using a freeze-pump-thaw method before use.

\section{Preparation of Eu-Alginate/PVA Hydrogel and Organohydrogels}

Eu-alginate/PVA hydrogel was prepared by following the method described in our previous work (Hu et al., 2017). Briefly, Na-alginate and PVA were dissolved in ultrapure water to produce a homogeneous solution, wherein the molar ratio of $\mathrm{Na}$-alginate and PVA is 1:9. The Na-alginate/PVA hydrogel was then obtained by two freeze/thaw cycles of the polymer solution. Subsequently, the Na-alginate/PVA hydrogel was soaked into the aqueous solution of $\mathrm{EuCl}_{3} \cdot 6 \mathrm{H}_{2} \mathrm{O}(0.01 \mathrm{~mol} / \mathrm{L})$, obtaining Eu-alginate/PVA hydrogel.

Eu-alginate/PVA organohydrogels were synthesized by using solvent displacement method (Chen et al., 2018). Herein, Eu-alginate/PVA hydrogel was directly placed into a vessel containing three different cryoprotectant solutions, namely ethylene glycol (EG), glycerol (GC) solution, and dsorbitol (SB) aqueous solution ( $\mathrm{SB}: \mathrm{H}_{2} \mathrm{O}=2: 1$ ), respectively. For the sake of brevity, we denote these Eu-alginate/PVA organohydrogels as $\mathrm{OHG}_{E G t}, \mathrm{OHG}_{G C t}$, and $\mathrm{OHG}_{S B t}$. OHG refers to organohydrogels, $\mathrm{EG}, \mathrm{GC}$, and $\mathrm{SB}$ denote the corresponding solution, and $t$ represents displacement time. To estimate the solvent displacement behaviors, the weight ratio $\left(\mathrm{W}_{\mathrm{a}} / \mathrm{W}_{\mathrm{b}}\right.$, where $\mathrm{W}_{\mathrm{b}}$ and $\mathrm{W}_{\mathrm{a}}$ refers to the weight before and after solvent displacement, respectively) of the organohydrogels was calculated. $\mathrm{OHG}_{0}$ means original Eu-alginate/PVA hydrogel. The synthesis procedures and structure of Eu-alginate/PVA OHG were illustrated in Figure 1.

\section{Measurement of Mechanical Properties}

All mechanical properties of the gels were tested on a tensile tester (CMT6503, MTS, United States). Tensile test was performed under room temperature, by setting a $500 \mathrm{~N}$ sensor. All the samples were cut into dumbbell-shaped with the help of a caliper in the size of tensile part $2 \times 2 \times 12 \mathrm{~mm}$ (Hengliang Liangju Co. Ltd., Shanghai, China). Both ends of the dumbbell-shaped 


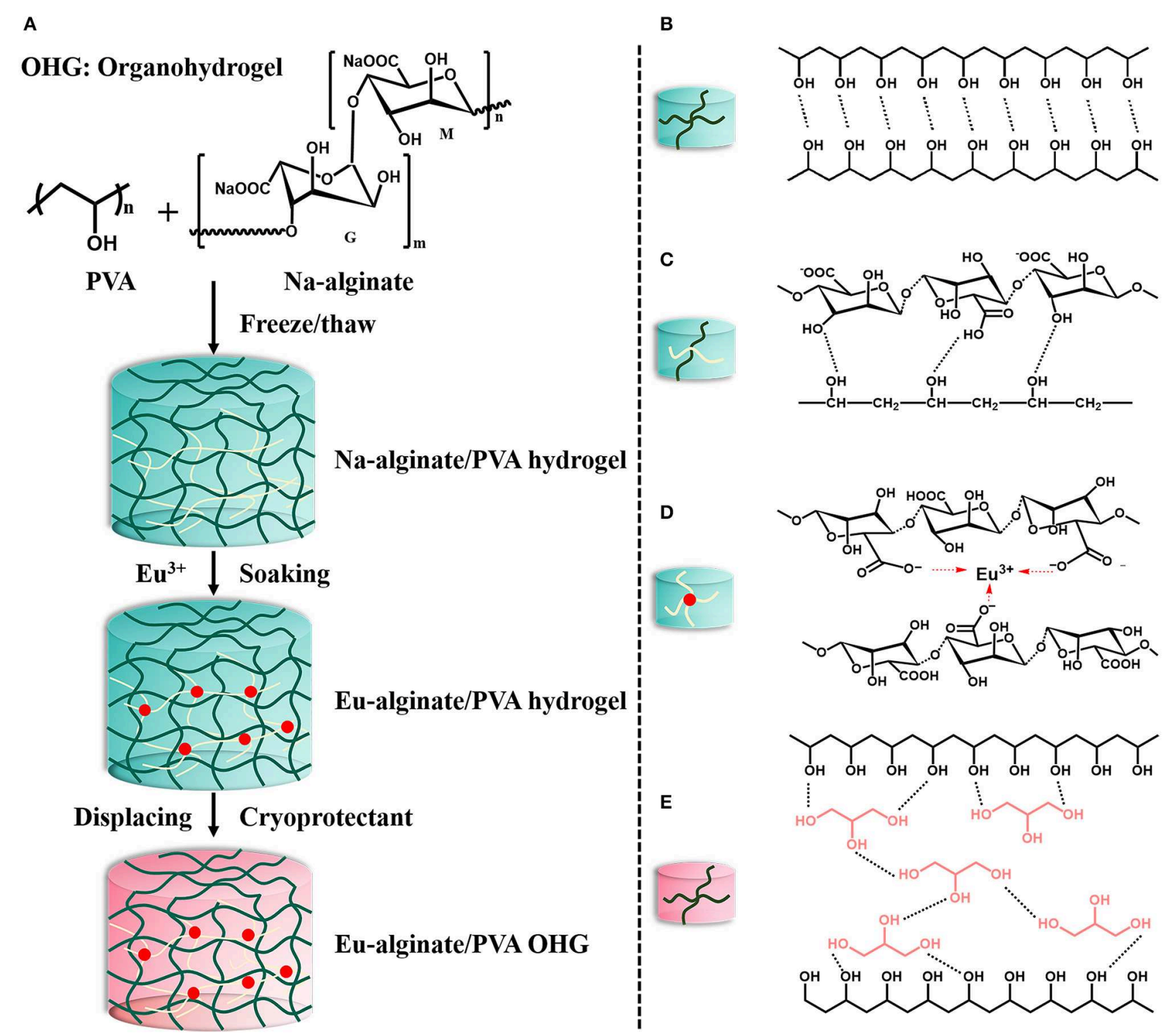

FIGURE 1 | The design strategy for physical organohydrogels with enhanced strength and extreme temperature tolerance via solvent displacement method. (A) Synthesis procedures and proposed structure of Eu-alginate/PVA organohydrogels. (B) Schematic illustration of hydrogen-bonding interactions of adjacent PVA polymers chains. (C) Hydrogen bonds between PVA polymers and Na-alginate macromolecules. (D) Anionic carboxyl groups in alginate structure coordination with $\mathrm{Eu}^{3+}$ ions (E). Hydrogen bonding between glycerol (GC) and $\mathrm{PVA}$ polymers in $\mathrm{OHG}_{\mathrm{GC}}$ obtained by solvent displacement method.

samples were connected to the clamps. The upper clamp was pulled by the load cell at a constant velocity of $100 \mathrm{~mm} \mathrm{~min}^{-1}$ while the lower clamp was fixed. From the stress-strain curve, the stress, Young's modulus and fracture strain of those gels can be calculated. The Young's modulus (E) could be calculated by $\tau=$ stress/strain when strain is lower than $10 \%$, where stress represents the force causing the deformation divided by the area to which the force is applied, and strain denotes the ratio of the change in elongation compared to the original length of the sample. Since strain is a dimensionless quantity, the unit of $\mathrm{E}$ is same as that of stress. Fracture strain is the maximum deformation tensile length that an object or substance can withstand, which can be calculated by $\varepsilon=\left(L-L_{0}\right) / L_{0}$, where $\mathrm{L}_{0}$ and $\mathrm{L}$ is the original and deformation length of the sample, respectively.

\section{Characterization of Non-drying and Anti-freezing Properties}

To gain further insight into the non-drying property of organohydrogels, the weight rate $\left(\mathrm{W}_{\mathrm{t}} / \mathrm{W}_{0}\right)$ was calculated. The initial weight of the sample was recorded as $\mathrm{W}_{0} \cdot \mathrm{W}_{\mathrm{t}}$ denotes the weight of the corresponding sample heated with different displacement times. The organohydrogels were heated at the temperature of $50^{\circ} \mathrm{C}$. Characterization of the anti-freezing 
property of organohydrogel was carried out by freezing the organohydrogels at the temperature of $-20^{\circ} \mathrm{C}$ or even $-45^{\circ} \mathrm{C}$ for $2 \mathrm{~h}$. The frozen organohydrogels were quickly folded or twisted and then left to recover freely. After $5 \mathrm{~min}$, the antifreezing property of the organohydrogels was illustrated in the digital pictures.

\section{Structural Characterization}

Characterization of the gels including morphology, composition, and crystalline structure, was carried out to further understand the solvent displacement mechanism. The morphology of the organohydrogel samples was visualized using a field-emission scanning electron microscopy (SEM, JROL JSM-7000F, Japan). Fourier transform infrared (FTIR) spectrum was collected at ambient temperature using a Nicolet 5700 FTIR spectrometer (Thermo Scientific, United States) over a wavelength ranges from 400 to $4,000 \mathrm{~cm}^{-1}$ after 64 scans at $2 \mathrm{~cm}^{-1}$ resolution. X-ray diffraction (XRD) patterns of the gels were obtained at room temperature on a Philips X'Pert pro MPD diffractometer, using $\mathrm{Cu}-\mathrm{K} \alpha$ radiation $(\lambda=1.5406 \AA)$ in the range of $2 \theta=10-$ $90^{\circ}$ and the scanning rate was set at $0.02^{\circ} / \mathrm{s}$. The hydrogel and organohydrogels were freeze-dried, before characterizing by the SEM and FTIR. The XRD results were directly obtained from the as-prepared hydrogel and organohydrogels.

\section{RESULTS AND DISCUSSIONS}

\section{Synthesis of the Eu-Alginate/PVA Organohydrogels}

The main synthetic procedures including three sequential steps to obtain the Eu-alginate/PVA organohydrogels were shown in Figure 1A. Firstly, homogeneous solution of PVA and Naalginate was freezed/thawed for two cycles to obtain $\mathrm{Na}$ alginate/PVA hydrogel. The procedure facilitates the formation of hydrogen bonds between the polymer chains in the Naalginate/PVA hydrogel. The hydrogen bonds formed between hydroxyl groups $(-\mathrm{OH})$ of PVA polymers (Figure 1B) as well as between carboxyl groups $(-\mathrm{COOH})$ of Na-alginate macromolecules and the hydroxyl groups of PVA polymers in the crosslinked nodes (Figure 1C). Subsequently, Na-alginate/PVA hydrogel was immersed in $\mathrm{EuCl}_{3}$ solution, and $\mathrm{Eu}^{3+}$ ions are easily accessible to anionic carboxyl groups in alginate structure center to form coordinate bonds (Figure 1D). $\mathrm{Eu}^{3+}$ ions, with low toxicity and antibacterial property, not only provide the photoluminescent property but also serve as physical crosslinkers for Na-alginate. Furthermore, coordination bonds between trivalent $\mathrm{Eu}^{3+}$ ions and the carboxyl ligands of $\mathrm{Na}$ alginate act as physical sacrificial bonds for energy dissipation, leading to good mechanical property. A tough Eu-alginate/PVA hydrogel could be obtained, and the hydrogel exhibits a dual physically crosslinked polymer networks including hydrogen bonds forming between polymer chains, as well as coordination bonds between $\mathrm{Eu}^{3+}$ ions and $-\mathrm{COO}^{-}$groups, while the dual crosslinked polymer networks endow tough mechanical behavior to the hydrogel (Hu et al., 2017).

Then, the Eu-alginate/PVA hydrogel was soaked into cryoprotectant solutions for a certain time to obtain organohydrogels swollen water-cryoprotectant binary solvent of EG, GC, and SB, respectively. Owing to osmotic pressure, a large amount of water in the hydrogel networks was displaced by cryoprotectants (bottom in Figure 1A). Furthermore, based on the principle of dissolution in a similar material structure, cryoprotectant molecules containing hydroxyl groups could be dispersed well in polymer networks to form hydrogen bonds with PVA polymer chains, obtaining the final extreme tough and temperature tolerant organohydrogels denoted as $\mathrm{OHG}_{E G}$, $\mathrm{OHG}_{G C}$, and $\mathrm{OHG}_{S B}$. We anticipate that the organohydrogels exhibit the superior mechanical properties in virtue of multiple hydrogen bonds between cryoprotectant molecules and PVA polymer chains (Figure 1E, taking $\mathrm{OHG}_{G C}$ as an example).

Figure 2 shows the weight rate $\left(\mathrm{W}_{\mathrm{a}} / \mathrm{W}_{\mathrm{b}}\right.$, where $\mathrm{W}_{\mathrm{b}}, \mathrm{W}_{\mathrm{a}}$ represents the weight before and after solvent displacement, respectively) of organohydrogels with the displacement time ranging from 0.5 to $6 \mathrm{~h}$. The weight rate is dependent on displacement time of the cryoprotectants. As can be seen, with increased displacement time, the weight rate of the organohydrogels decreased and finally almost reached a displacement equilibrium state. Especially, the weight rate $\left(\mathrm{W}_{\mathrm{a}} / \mathrm{W}_{\mathrm{b}}\right)$ of $\mathrm{OHG}_{E G}, \mathrm{OHG}_{G C}$, and $\mathrm{OHG}_{S B}$ all decreased quickly at initial $0.5 \mathrm{~h}$, from 1 to $0.55,0.63$, and 0.58 , respectively (Figure 2A). The water in the hydrogel system usually exists in three states, i.e., free water, intermediate water, and nonrotational bound water (Cerveny et al., 2005; Wu et al., 2019). The fastdecreased weight rate is attributed to the fact that most of the "free water" in hydrogel networks is displaced quickly due to unbound water molecules. However, the intermediate water weakly interacted with the polymer networks is displaced slowly with the cryoprotectant molecules. Moreover, it is difficult for the strongly bound water to undergo solvent displacement. As an example, with the displacement time ranging from 0 to $6 \mathrm{~h}$, the fast shrank and decreased volume of the ethylene glycol based organohydrogel $\left(\mathrm{OHG}_{E G}\right)$ could be visualized at initial time $(<0.5 \mathrm{~h})$ and then it slowed down (Figure 2B). These results indicate a successful solvent displacement between the water and cryoprotectant molecules. With this approach, the Eu-alginate/PVA hydrogel was transformed into Eualginate/PVA organohydrogels with dense polymer networks swollen water-cryoprotectant binary solvent, leading to enhanced capabilities of mechanical properties, moisture holding and temperature tolerance.

\section{Mechanical Properties of the Eu-Alginate/PVA Organohydrogels}

The effects of cryoprotectants on the mechanical properties of organohydrogels were tested by tensile experiments. Figure 3 shows the mechanical properties (tensile strength, fracture strain and Young's modulus) of the organohydrogels $\left(\mathrm{OHG}_{E G}, \mathrm{OHG}_{G C}\right.$, and $\mathrm{OHG}_{S B}$ ) displaced by three different cryoprotectants. The tensile strength (Figure 3B), fracture strain (Figure 3C), and Young's modulus (Figure 3D) of the organohydrogels were higher than that of original Eu-alginate/PVA hydrogel, which can be ascribed to the synergistic effect of the multiple hydrogen bonds and the dense polymer networks. For instance, the tensile 

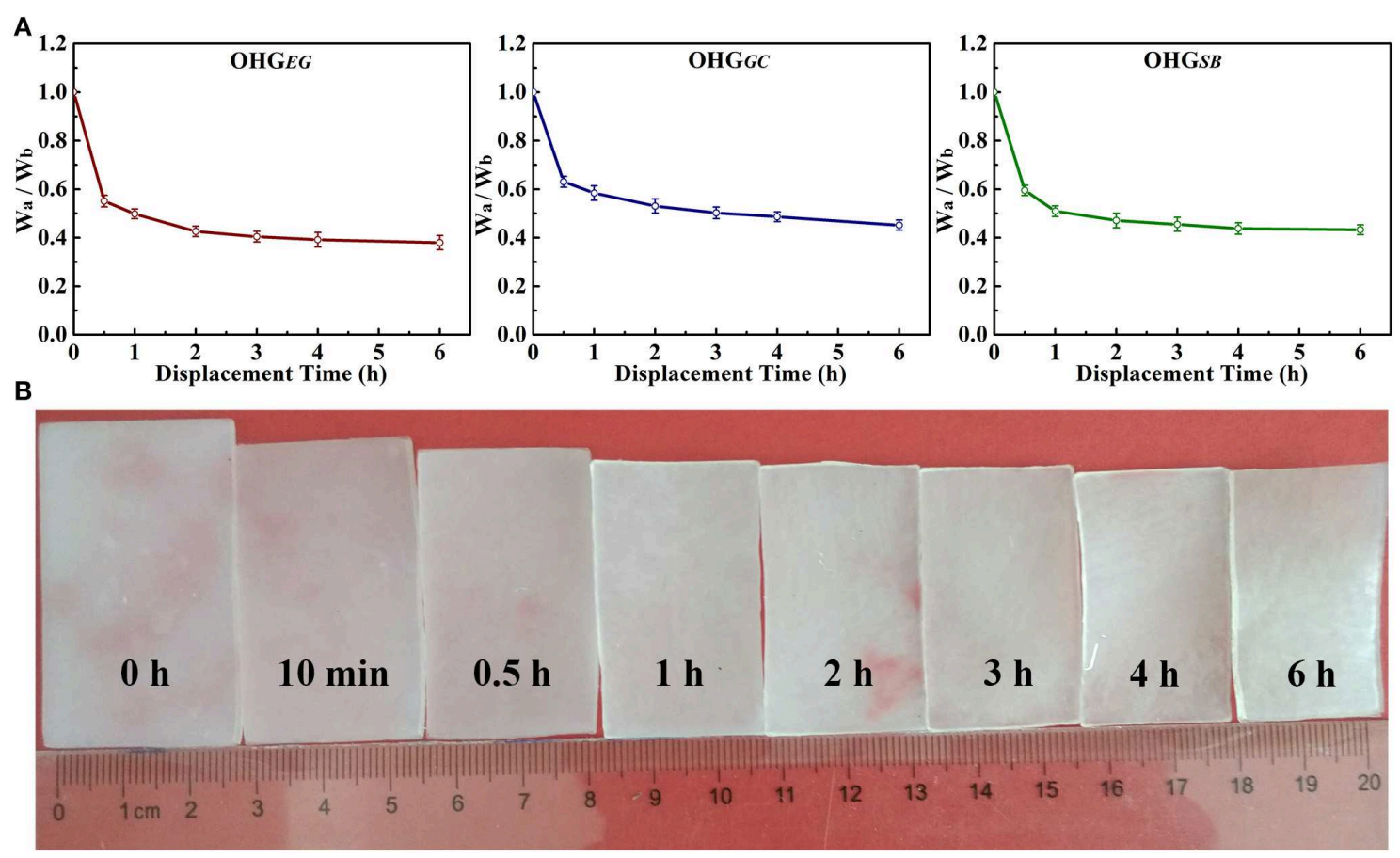

FIGURE 2 | Weight rate $\left(W_{a} W_{b}\right)$ of the organohydrogels (A). $W_{a}$ denotes the weight of the gel immersed in different cryoprotectant solution [ethylene glycol (EG), glycerol (GC), and d-sorbitol (SB) solutions] with different displacement time. $W_{b}$ represents the original weight of the Eu-alginate/PVA hydrogel. (B) Digital pictures show change in the size of $\mathrm{OHG}_{E G}$ with prolonged displacement time of $0 \mathrm{~h}, 10 \mathrm{~min}, 0.5 \mathrm{~h}, 1 \mathrm{~h}, 2 \mathrm{~h}, 3 \mathrm{~h}, 4 \mathrm{~h}$ and $6 \mathrm{~h}$.

strength of $\mathrm{OHG}_{S B}$ increases from $0.58 \pm 0.06 \mathrm{MPa}$ to as high as $5.62 \pm 0.41 \mathrm{MPa}$, fracture strain raised from $4.07 \pm 0.04$ to as high as $7.63 \pm 0.02$ and Young's modulus ascended from $0.16 \pm 0.01$ to $1.08 \pm 0.03 \mathrm{MPa}$ as the displacement time gradually increased to $6 \mathrm{~h}$. The tensile strength is higher than many of the previously reported organohydrogels, such as polydopamine decorating carbon nanotubes (PDA-CNT)/copolymer of acrylamide (AM) and acrylic acid (AA) (PAM-co-PAA) organohydrogel $(0.07$ MPa stress, 7.01 strain, Han et al., 2017), PVA/poly(3,4ethylenedioxythiophene):polystryrene sulfonate (PEDOT:PSS) organohydrogel (2.1 MPa stress, 7.60 strain, Rong et al., 2017), and gelation organohydrogel (2.06 MPa stress, 6.88 strain, Qin et al., 2019), as shown in Figure S1. The dramatic enhancement in mechanical properties of the organohydrogels is directly related to crosslinking density, which dominated by the largely increased hydrogen bonds between the cryoprotectant molecules and polymer chains in the organohydrogels (Pan et al., 2018). Interestingly, the tensile strength and the Young's modulus of $\mathrm{OHG}_{\mathrm{EG}}$ and $\mathrm{OHG}_{\mathrm{GC}}$ increased by increasing displacement time and then its tended to balance. The tensile strength of $\mathrm{OHG}_{E G}$ and $\mathrm{OHG}_{G C}$ prepared at displacement time of 3 and $4 \mathrm{~h}$ reached to $3.20 \pm 0.37$ and $3.45 \pm 0.42 \mathrm{MPa}$, respectively. The Young's modulus of the organohydrogels reached to 0.98 \pm 0.34 and $0.99 \pm 0.42 \mathrm{MPa}$, respectively. The excellent tensile strength and Young's modulus achieved in the shorter displacement time could be attributed to the smaller molecules of EG and GC than SB. Overall, based on solvent displacement method, the mechanical strength of the physically crosslinked organohydrogels can be dramatically enhanced. In addition, the types of cryoprotectants and displacement time play important roles in controlling mechanical performances of organohydrogels to fulfill the requirements in specific potential applications.

\section{The Non-drying and Anti-freezing Properties of the Eu-Alginate/PVA Organohydrogels}

To demonstrate the organohydrogels with a temperature tolerance $\left(-45-50^{\circ} \mathrm{C}\right)$, we investigated the non-drying and antifreezing properties of the organohydrogels $\left(\mathrm{OHG}_{E G}, \mathrm{OHG}_{G C}\right.$, $\mathrm{OHG}_{S B}$ ), as shown in Figure 4. Firstly, to demonstrate nondrying property, the organohydrogels immersed in EG, GC, and SB for different time $(0-6 \mathrm{~h})$ were heated at the temperature of $50^{\circ} \mathrm{C}(0-13 \mathrm{~h})$. The weight rate was calculated by $\left(\mathrm{W}_{\mathrm{t}} / \mathrm{W}_{0}\right)$, where $\mathrm{W}_{0}$ and $\mathrm{W}_{\mathrm{t}}$ denotes for the weight of organohydrogels before heating and heating for certain time, respectively (Figure 4B). The weight of organohydrogels decreased by increasing heating time and then it tended to balance, due to that remaining water was evaporated from the organohydrogels. Furthermore, the organohydrogels treated with long displacement time showed high weight rate $\left(\mathrm{W}_{\mathrm{t}} / \mathrm{W}_{0}\right)$. Notably, it was found that the weight rate of the $\mathrm{OHG}_{G C}$ at the displacement time of $6 \mathrm{~h}$ exhibited the highest weight rate (over 0.9 ), because lower vapor pressure (compared to glycol) and fast exchange kinetics of glycerol (small molecular size compared to sorbitol) (Rajan and Matsumura, 2018). In contrast, the original hydrogel showed the lowest weight 

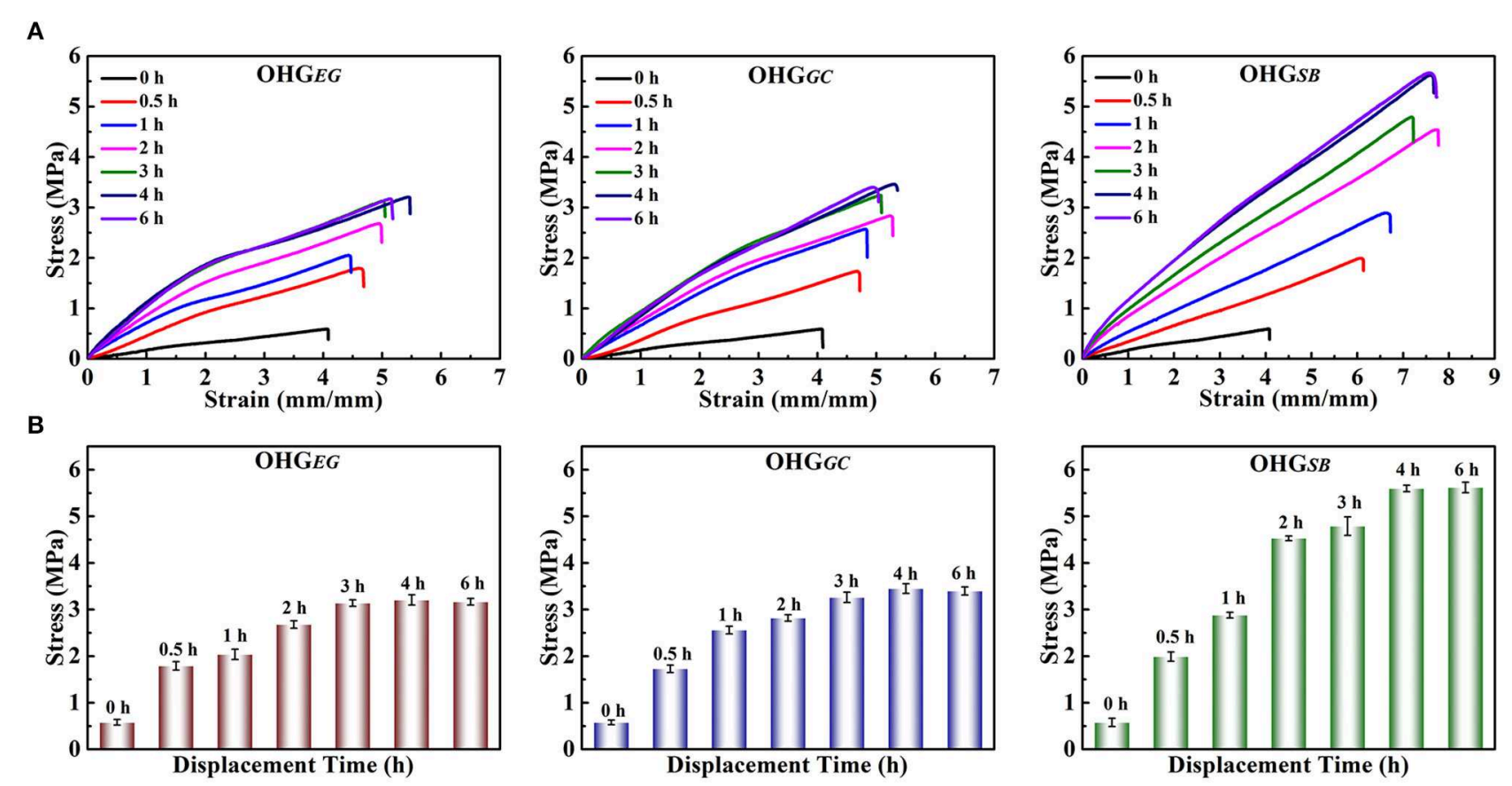

C
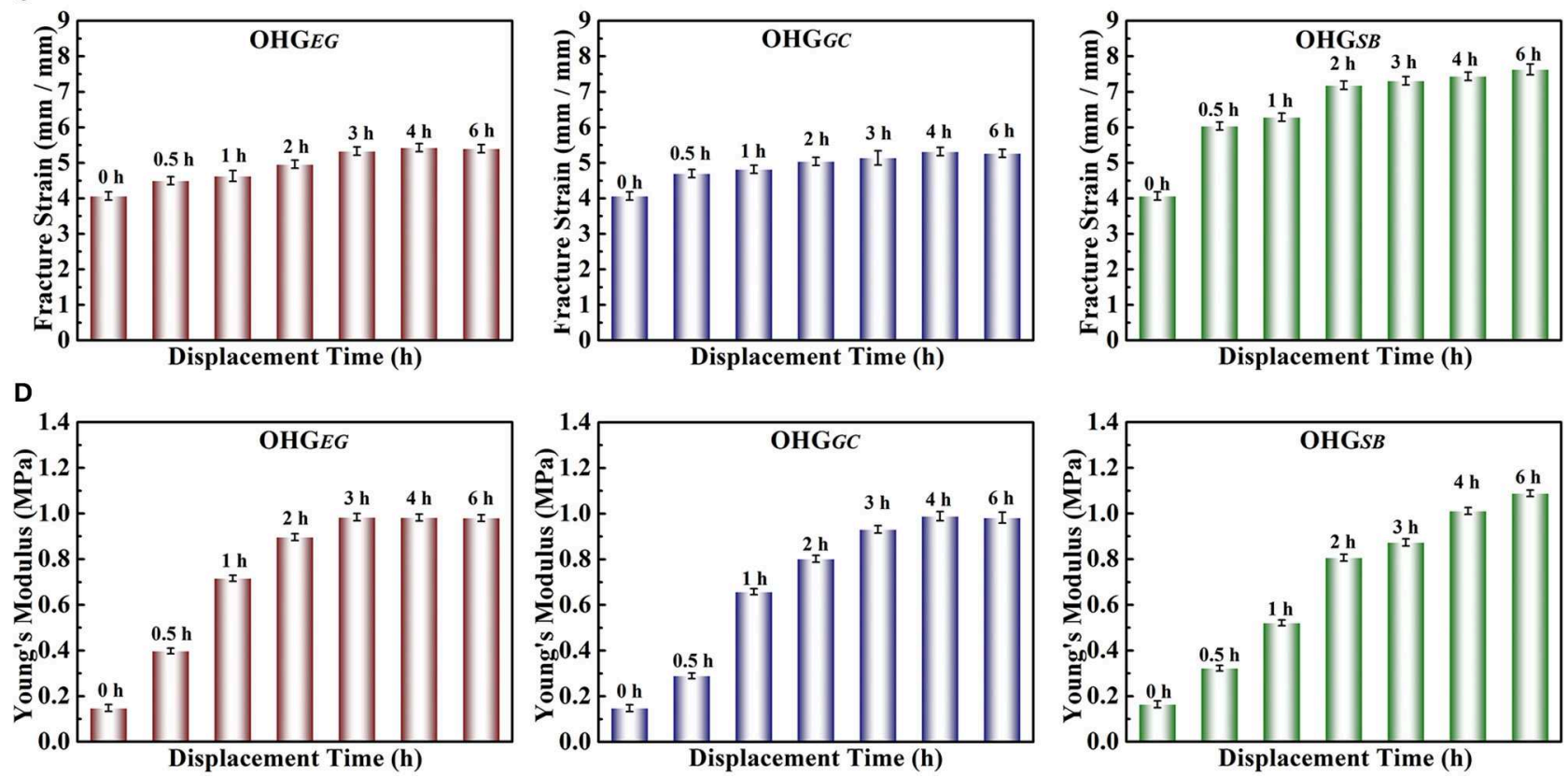

FIGURE 3 | Mechanical properties of the Eu-alginate/PVA organohydrogels (OHGEG, OHG $\mathrm{OHC}_{\mathrm{G}}$ OHGs) treated with cryoprotectants for different times. (A) stress-strain curves, (B) stress-displacement time histogram, (C) Fracture strain-displacement time histogram, (D) Young's modulus-displacement time histogram.

rate (0.18), indicating that $\mathrm{OHG}_{0}$ does not show non-drying ability due to volatilization of water.

In addition, the anti-freezing properties of the organohydrogels, i.e., deformation behaviors (c, bend; d, twist for $3 \times 360^{\circ}$; e, fold) and corresponding recovery states were demonstrated in Figure 4. The behaviors of the organohydrogels and hydrogel under the sub-zero temperature were obviously different. The organohydrogels exhibited outstanding deformation behavior, but the original hydrogel could not recover after bending under the sub-zero temperature. The frozen hydrogel $\left(\mathrm{OHG}_{0}\right)$ displayed a non-transparent and white morphology due to formation of an aggregate of ice crystals in the polymer networks (Figure 4C). The organohydrogels displaced by different cryoprotectants for $4 \mathrm{~h}$, 


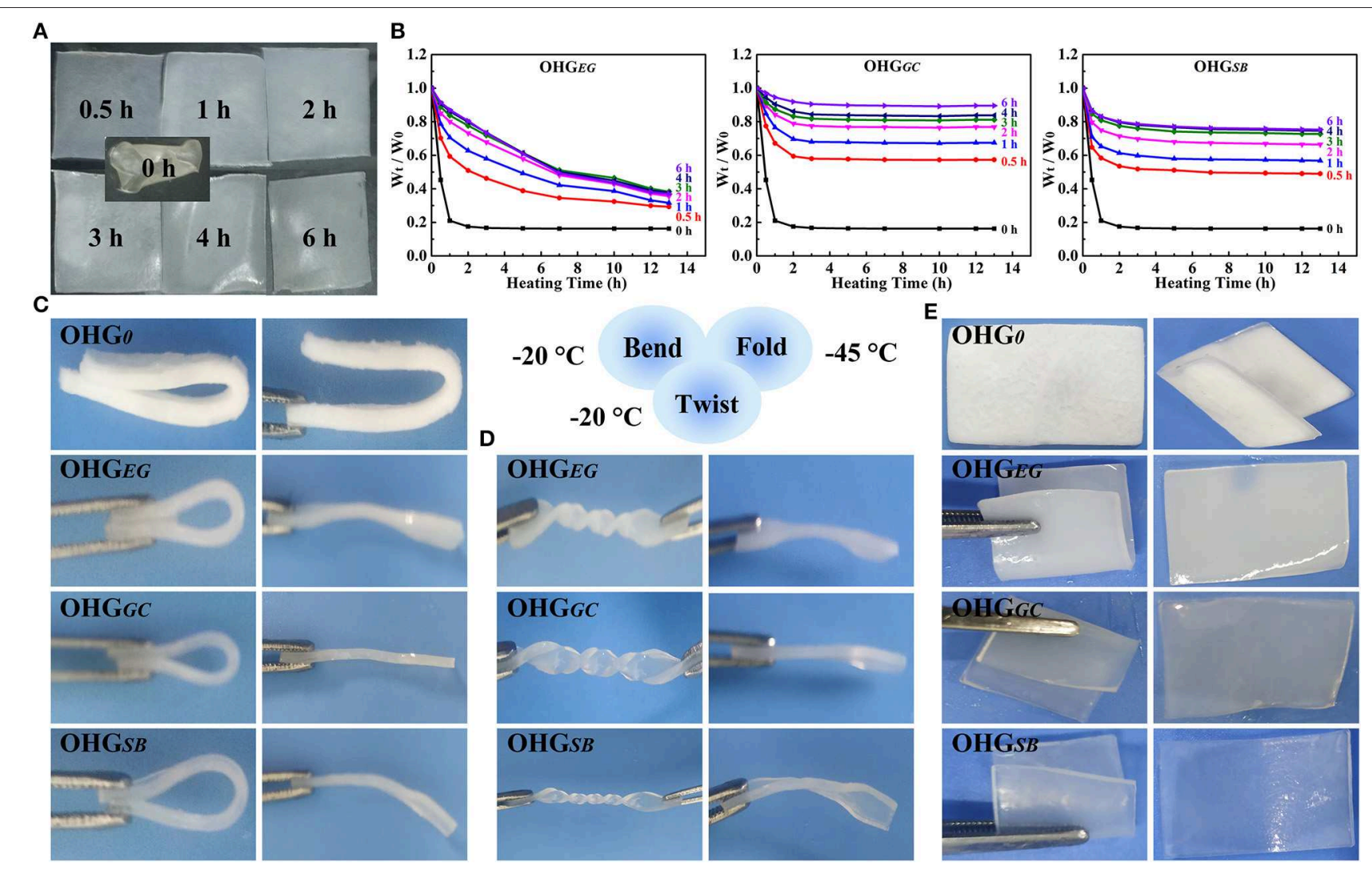

FIGURE 4 | Non-drying (A,B) and anti-freezing (C-E) properties of the organohydrogels. (A) Digital pictures of the organohydrogels soaked in GC solution for different times $(0,0.5,1,2,3,4,6 \mathrm{~h})$ and then heated in a vacuum oven at $50^{\circ} \mathrm{C}$ until constant weight was gained. (B) Weight retention rate of $\mathrm{OHG}$. $\mathrm{OHG}_{G C}$, and $\mathrm{OH}_{S B}$ with different immersion time under heating at $50^{\circ} \mathrm{C}$ for different heating time. $W_{0}$ is the initial weight of the organohydrogels $\left(\mathrm{OHG}_{0}\right)$, while $W_{\mathrm{t}}$ denotes the weight of the samples heated for different times at the temperature of $50^{\circ} \mathrm{C}$. The mechanical deformation behaviors $\left(\mathbf{C}\right.$, bent; $\mathbf{D}$, twisted for $3 \times 360^{\circ}$; $\mathbf{E}$, fold) and corresponding recovery state (after free recovery for $5 \mathrm{~min}$ ) of the organohydrogels soaked for $4 \mathrm{~h}$ and then cooled at $-20^{\circ} \mathrm{C}(\mathbf{C}, \mathbf{D})$ and $-45^{\circ} \mathbf{C}(\mathbf{E})$.

for example, $\mathrm{OHG}_{E G 4}$, $\mathrm{OHG}_{G C 4}$, and $\mathrm{OHG}_{S B 4}$, showed good recovery behaviors after being bent and twisted for $3 \times 360^{\circ}$ at $-20^{\circ} \mathrm{C}$ (Figure $4 \mathrm{D}$ ). To further demonstrate the anti-freezing property of the organohydrogels, the organohydrogels and hydrogel were placed in a harsh condition $\left(-45^{\circ} \mathrm{C}\right)$. In fact, the hydrogel became rigid and fragile owing to being frozen and even generated cracks on the surface during folding under the subzero temperature. In contrast, the organohydrogels $\left(\mathrm{OHG}_{E G 4}\right.$, $\mathrm{OHG}_{G C 4}$, and $\mathrm{OHG}_{S B 4}$ ) could return to their initial states after being bent and folded (Figure 4E). The excellent anti-freezing ability is due to the ice-inhibiting effect of cryoprotectants disrupting the formation of ice crystal lattices of the residual water molecules. The results demonstrate that the cryoprotectant based organohydrogels exhibit excellent non-drying and antifreezing property, indicating the potential applications under a broad temperature range.

\section{The Microstructure of Organohydrogels and Hydrogel}

To further demonstrate the effect of microstructural changes of organohydrogels and hydrogel on their performances, the SEM, XRD, and FTIR analyses were performed, respectively.
As shown in the SEM images (Figure 5A), the original hydrogel $\left(\mathrm{OHG}_{0}\right)$ displayed a distinct porous structure with loose texture, because water molecules form a lot of ice crystals under subzero temperature, and leading to porous structure mainly occupied by water after sublimation of ice crystals from the hydrogel processed by vacuum freeze-drying (Ricciardi et al., 2004). On the other hand, organohydrogels

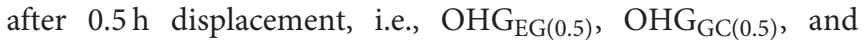
$\mathrm{OHG}_{\mathrm{SB}(0.5)}$ presents dense structure after same treatment processes, because cryoprotectants prevent formation of ice crystals. The organohydrogels with dense structure correspond to volume shrinkage of organohydrogels because hydrophilic polymers do not swell well in the cryoprotectants (Figure 2).

Additionally, the unique microstructures could strengthen the crystallization among PVA polymer chains. It could be verified by XRD patterns where the crystal peak of PVA became more intense in the organohydrogels $\left(\mathrm{OHG}_{E G(0.5)}, \mathrm{OHG}_{G C(0.5)}\right.$, and $\mathrm{OHG}_{S B(0.5)}$ ), than that of hydrogels (Figure 5B). The Eualginate/PVA hydrogel and organohydrogels do not show sharp crystalline diffraction peaks of PVA, because the presence of $\mathrm{Na}$-alginate and $\mathrm{Eu}^{3+}$ ions inhibit the crystallization of PVA (Hu et al., 2017). The Eu-alginate/PVA hydrogel has a halo 

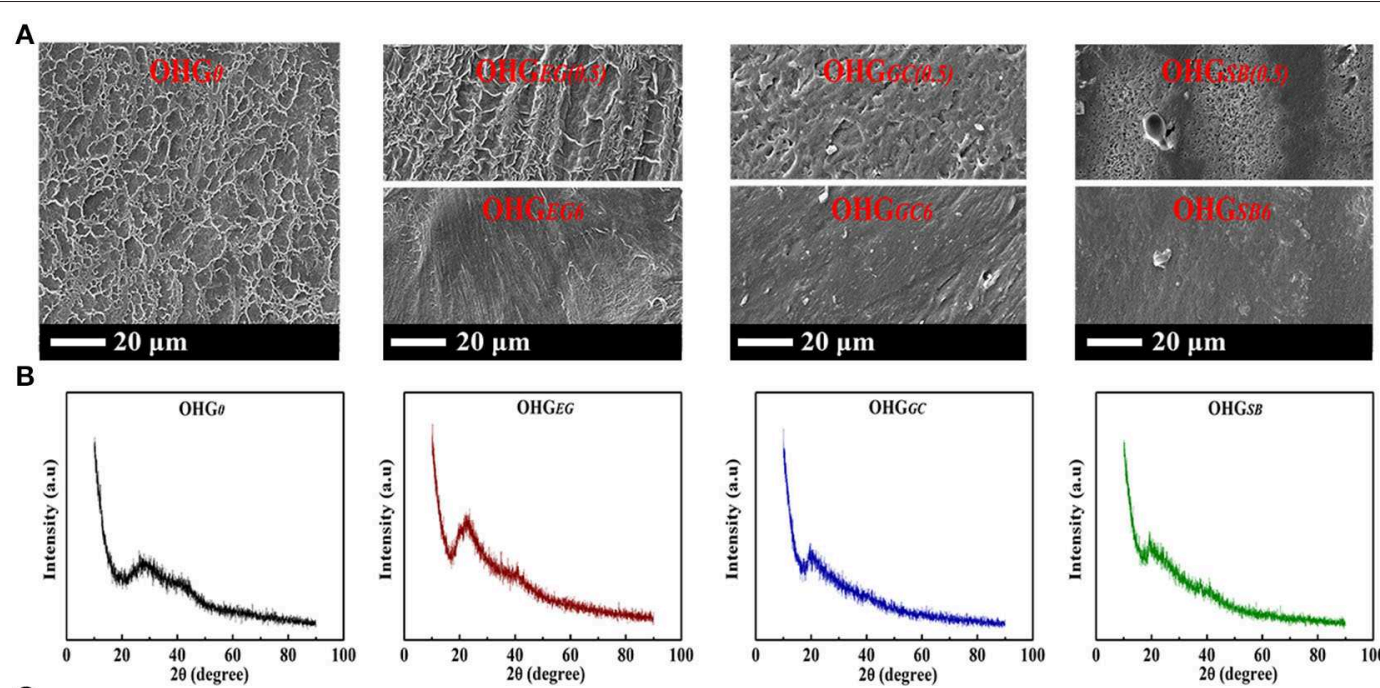

C
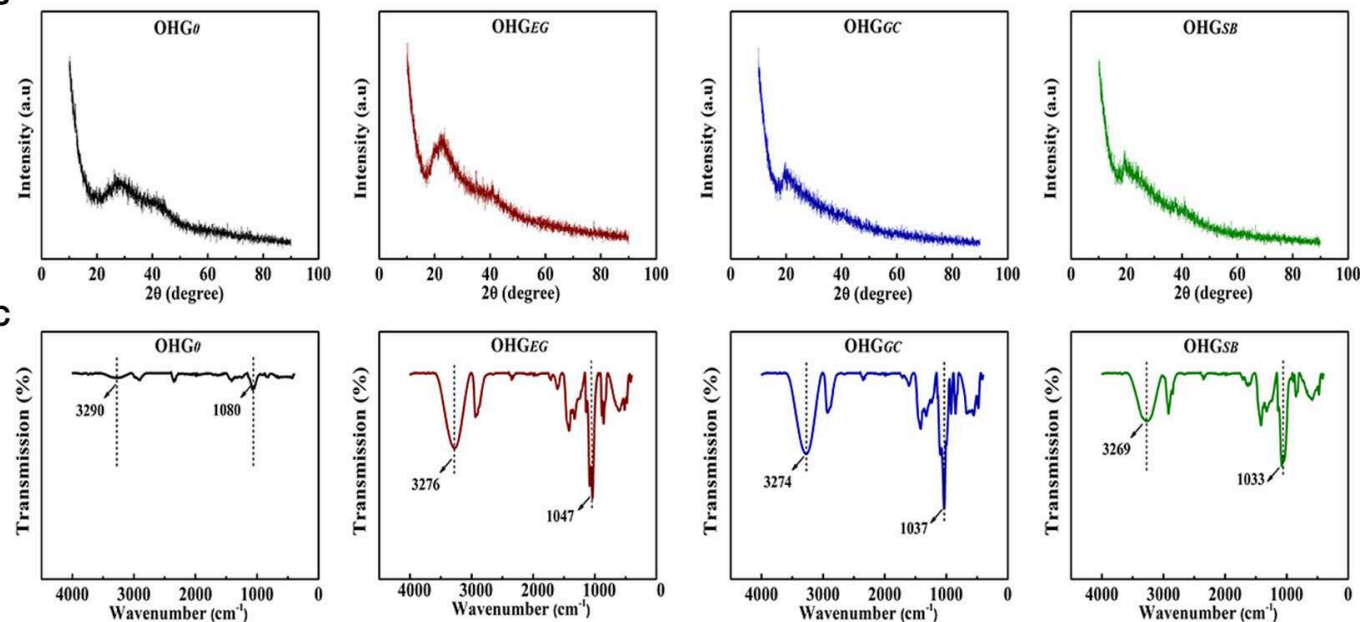

D

OHGo
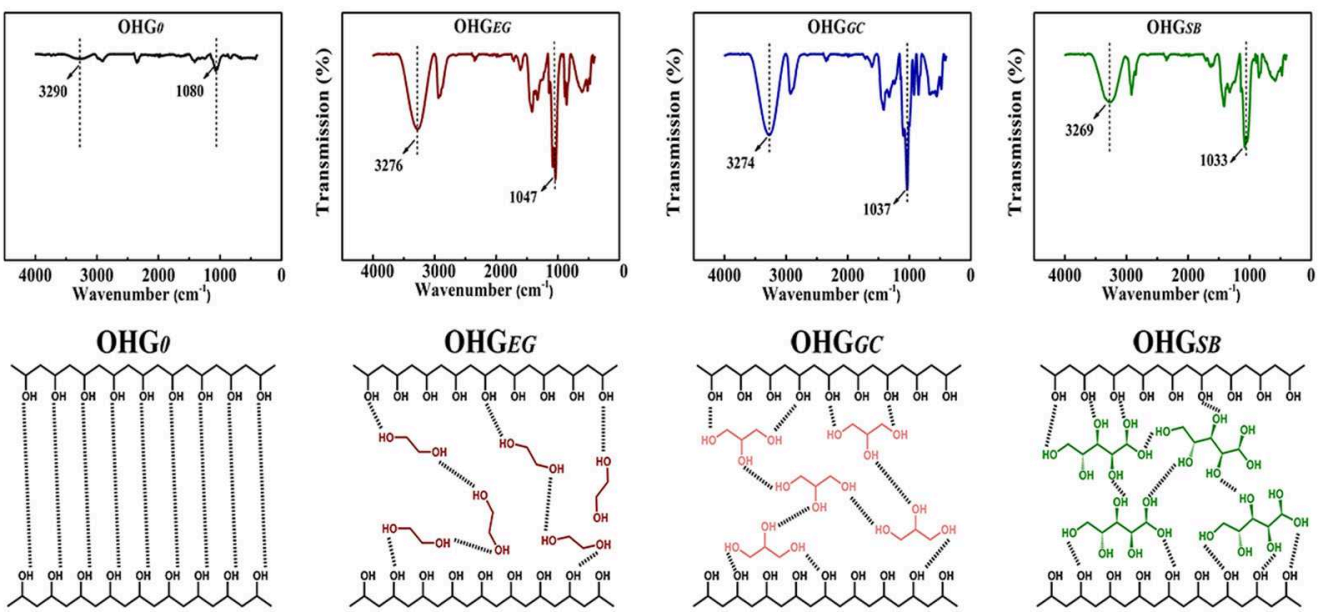

OHGSB

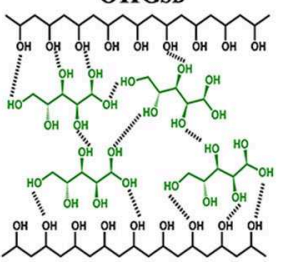

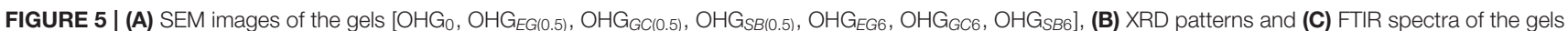
$\left(\mathrm{OHG}_{0}, \mathrm{OHG}_{E G 6}, \mathrm{OHG}_{G C 6}, \mathrm{OHG}_{S B 6}\right)$. For example, $\mathrm{OHG}_{E G(0.5)}$ represents Eu-alginate/PVA hydrogels soaking in ethylene glycol (EG) for $0.5 \mathrm{~h}$, while $\mathrm{OHG} \mathrm{G}_{S 6}$ denotes the hydrogels soaking in d-sorbitol (SB) for $6 \mathrm{~h}$. (D) Schematic illustrations of the hydrogen bonds (black dotted line) between PVA polymer chains in hydrogel (OHG), and EG, GC, SB molecules bridged PVA chains via forming hydrogen bonds in the corresponding organohydrogels.

centered at $2 \theta \approx 28^{\circ}$ (Figure $5 \mathbf{B}$ ) in the diffraction of pure water same as previous report (Ricciardi et al., 2004). The Eualginate/PVA hydrogel possesses a water content high enough to $85 \%$ while a low PVA content of about $13.5 \%$, indicating crystalline diffraction peaks of PVA $\left(2 \theta \approx 19.4\right.$ and $\left.20^{\circ}\right)$ might be covered by the aforementioned diffraction of free pure water. After the hydrogel was transformed into organohydrogels, typical reflections of crystalline atactic PVA, with a maximum $2 \theta$ angles of $22.3,20.1,19.6^{\circ}$ presents for $\mathrm{OHG}_{\mathrm{EG}}, \mathrm{OHG}_{\mathrm{GC}}$, and $\mathrm{OHG} \mathrm{GB}_{\mathrm{SB}}$ sample, respectively (Ricciardi et al., 2004). A slight shift of the peak around $2 \theta=20.0^{\circ}$ of three type organohydrogels could be assigned to the hydrogen bonds between PVA polymers and the cryoprotectants with different molecular structures (Zhao et al., 2019). The results indicate more crystalline PVA aggregates are formed in the organohydrogels due to the decreased relative amount of "free water," whereas a lot of swollen amorphous PVA polymer chains present in the hydrogel. The multiple hydrogen bonds including PVA crystalline domains act as knots of the gel network, promoting the enhancement of mechanical properties (Figure 5D).

As shown in the FTIR spectrums (Figure 5C), the FTIR spectrum of Eu-alginate/PVA hydrogel shows the characteristic stretching bands of $-\mathrm{OH}$ at $3,290 \mathrm{~cm}^{-1}$ and $\mathrm{C}-\mathrm{O}$ at $1,080 \mathrm{~cm}^{-1}$. While for the Eu-alginate/PVA organohydrogels $\left(\mathrm{OHG}_{\mathrm{EG}}\right.$, $\mathrm{OHG}_{\mathrm{GC}}$ and $\mathrm{OHG}_{\mathrm{SB}}$ ), the characteristic stretching band of $\mathrm{OH}$ shifted to $3,276,3,274$, and $3,269 \mathrm{~cm}^{-1}$, respectively, as well as the characteristic stretching band of $\mathrm{C}-\mathrm{O}$ shifted to 1,047 , 1,037 , and $1,033 \mathrm{~cm}^{-1}$, respectively. The shift of IR absorption bands to lower wave numbers suggests the formation of stronger $\mathrm{H}$-bonding in the organohydrogels.

Based on the above analysis, schematics illustrating the interaction among PVA polymer chains and cryoprotectant molecules were presented in Figure 5D. After cryoprotectant displacement, the EG, GC, SB molecules could bridge PVA chains via abundant hydrogen bonds forming between cryoprotectants and PVA polymer chains. And the pivotal 
roles of cryoprotectant molecules can be attributed to three parts, that are, (i) Enhancing the mechanical properties of organohydrogels. (ii) Restricting volatilization of the residual water to promote non-drying ability. (iii) Disrupting the formation of ice crystal lattices as well as reducing the freezing point of $\mathrm{H}_{2} \mathrm{O}$, both phenomena increase the anti-freezing capacity of organohydrogels. As a result, physically crosslinked organohydrogels with enhanced mechanical properties and extreme temperature tolerance could be designed and obtained by cryoprotectants displacement method.

\section{CONCLUSIONS}

In summary, physically crosslinked organohydrogels with toughness and extreme temperature tolerance were successfully fabricated by solvent displacement method. Each component of the Eu-alginate/PVA organohydrogels serves the respective role for endowing excellent integrated properties. The PVA is responsible for gel backbone, offering a certain mechanical strength facilitating hydrogen bonds formation, while alginate enables the enhanced mechanical performance of the gels by coordination with $\mathrm{Eu}^{3+}$ ions, and interlaces with PVA polymer chains via hydrogen bonds. More importantly, cryoprotectants disrupt the formation of ice crystal lattices of water molecules. This disruption is responsible for bridging of PVA chains through abundant and stable multiple hydrogen bonds, profiting effective energy dissipation, and restricting volatilization of the residual water. The organohydrogels feature enhanced and tunable mechanical capacity, as well as freezing/heating tolerance, potentially to be used in various fields, such as medical devices, flexible electronics, and stretchable devices.

\section{REFERENCES}

Banerjee, H., Suhail, M., and Ren, H. (2018). Hydrogel actuators and sensors for biomedical soft robots: brief overview with impending challenges. Biomimetics 3:15. doi: 10.3390/biomimetics3030015

Cerveny, S., Colmenero, J., and Alegría, A. (2005). Dielectric investigation of the low-temperature water dynamics in the poly(vinyl methyl ether) $/ \mathrm{H}_{2} \mathrm{O}$ system. Macromolecules 38, 7056-7063. doi: 10.1021/ma050811t

Chen, F., Zhou, D., Wang, J., Li, T., Zhou, X., Gan, T., et al. (2018). Rational fabrication of anti-freezing non-drying tough organohydrogels by one-pot solvent displacement. Angew. Chem. Int. Ed. 130, 6568-6571. doi: 10.1002/ange.201803366

Chen, Q., Zhu, L., Chen, H., Yan, H., and Huang, L. (2015). A novel design strategy for fully physically linked double network hydrogels with tough, fatigue resistant, and self-healing properties. Adv. Funct. Mater. 25, 1598-1607. doi: 10.1002/adfm.201404357

Dhivya, S., Saravanan, S., Sastry, T. P., and Selvamurugan, N. (2015). Nanohydroxyapatite-reinforced chitosan composite hydrogel for bone tissue repair in vitro and in vivo. J. Nanobiotechnol. 13:40. doi: 10.1186/s12951-015-0099-Z

Dong, D., Hao, T., Wang, C., Zhang, Y., Qin, Z., Yang, B., et al. (2018). Zwitterionic starch-based hydrogel for the expansion and "stemness" maintenance of brown adipose derived stem cells. Biomaterials 157, 149-160. doi: 10.1016/j.biomaterials.2017.12.011

Elliott, G. D., Wang, S., and Fuller, B. J. (2017). Cryoprotectants: a review of the actions and applications of cryoprotective solutes that modulate cell recovery from ultra-low temperatures. Cryobiology 76, 74-91. doi: 10.1016/j.cryobiol.2017.04.004

\section{DATA AVAILABILITY STATEMENT}

All datasets generated for this study are included in the article/Supplementary Material.

\section{AUTHOR CONTRIBUTIONS}

YC put forward the ideas about this research and designed the experiments. JZ, DD, and EZ prepared the main materials, and completed the structural characterization and performance testing of materials. JZ and DD analyzed the data and wrote the manuscript. XG, YZ, KY, MK, YAr, and YAz revised and edited the manuscript.

\section{FUNDING}

This work was supported by the National Natural Science Foundation of China (11674263), Key Research and Development Program of Shaanxi (Program No. 2020KWZ006). Xi'an Weiyang District Science and Technology Fund (201927), Key Intergovernmental Special Projects of the National Key R \& D Plan of the People's Republic of China (No. 2018YFE0114200), the research project grant of the joint logistic support force (BLB18J014).

\section{SUPPLEMENTARY MATERIAL}

The Supplementary Material for this article can be found online at: https://www.frontiersin.org/articles/10.3389/fchem. 2020.00102/full\#supplementary-material

Fan, D., Tian, Y., and Liu, Z. (2019). Injectable hydrogels for localized cancer therapy. Front. Chem. 7:675. doi: 10.3389/fchem.2019.00675

Gao, H., Zhao, Z., Cai, Y., Zhou, J., Hua, W., Chen, L., et al. (2017). Adaptive and freeze-tolerant heteronetwork organohydrogels with enhanced mechanical stability over a wide temperature range. Nat. Commun. 8:15911. doi: 10.1038/ncomms15911

Gao, Y., Song, J., Li, S., Elowsky, C., Zhou, Y., Ducharme, S., et al. (2016). Hydrogel microphone for stealthy underwater listening. Nat. Commun. 24:13114. doi: $10.1038 /$ ncomms 13114

GhavamiNejad, A., SamariKhalaj, M., Aguilar, L. E., Park, C. H., and Kim, C. S. (2016). pH/NIR light-controlled multidrug release via a mussel-inspired nanocomposite hydrogel for chemo-photothermal cancer therapy. Sci. Rep. 6:33594. doi: 10.1038/srep33594

Gong, J. P. (2014). Materials both tough and soft. Science 344, 161-162. doi: $10.1126 /$ science. 1252389

Gong, J. P., Katsuyama, Y., Kurokawa, T., and Osada, Y. (2003). Double-network hydrogels with extremely high mechanical strength. Adv. Mater. 15, 1155-1158. doi: 10.1002/adma.200304907

Gong, Z., Zhang, G., Zeng, X., Li, J., Li, G., Huang, W., et al. (2016). Highstrength, tough, fatigue resistant, and self-healing hydrogel based on dual physically cross-linked network. ACS Appl. Mater. Interfaces 8, 24030-24037. doi: 10.1021/acsami.6b05627

Gu, B., Sun, X., Papadimitrakopoulos, F., and Burgess, D. J. (2016). Seeing is believing, PLGA microsphere degradation revealed in PLGA microsphere/PVA hydrogel composites. J. Control. Release 228, 170-178. doi: 10.1016/j.jconrel.2016.03.011

Han, J., Zhao, X., Xu, W., Wang, W., Han, Y., and Feng, X. (2018). Effect of hydrophobic polypeptide length on performances of 
thermo-sensitive hydrogels. Molecules 23:1017. doi: 10.3390/molecules230 51017

Han, L., Liu, K., Wang, M., Wang, K., Fang, L., Chen, H., et al. (2017). Musselinspired adhesive and conductive hydrogel with long-lasting moisture and extreme temperature tolerance. Adv. Funct. Mater. 28:1704195. doi: 10.1002/adfm.201704195

Han, Y., Bai, T., Liu, Y., Zhai, X., and Liu, W. (2012). Zinc ion uniquely induced triple shape memory effect of dipole-dipole reinforced ultrahigh strength hydrogels. Macromol. Rapid Commun. 33, 225-231. doi: $10.1002 /$ marc.201100683

Haraguchi, K., and Takehisa, T. (2002). Nanocomposite hydrogels: a unique organic-inorganic network structure with extraordinary mechanical, optical, and swelling/de-swelling properties. Adv. Mater. 14, 1120-1124. doi: 10.1002/1521-4095(20020816)14:163.0.CO;2-9

Hu, C., Wang, M. X., Sun, L., Yang, J. H., Zrínyi, M., and Chen, Y. M. (2017). Dual-physical cross-linked tough and photoluminescent hydrogels with good biocompatibility and antibacterial activity. Macromol. Rapid Commun. 38:1600788. doi: 10.1002/marc.201600788

Hu, X., Gao, Z., Tan, H., Wang, H., Mao, X., and Pang, J. (2019). An injectable hyaluronic acid-based composite hydrogel by DA click chemistry with $\mathrm{pH}$ sensitive nanoparticle for biomedical application. Front. Chem. 7:477. doi: 10.3389/fchem.2019.00477

Huang, T., Xu, H., Jiao, K., Zhu, L., Brown, H., Wang, H. A., et al. (2007). A novel hydrogel with high mechanical strength: a macromolecular microsphere composite hydrogel. Adv. Mater. 19, 1622-1626. doi: 10.1002/adma.2006 02533

Iwaso, K., Takashima, Y., and Harada, A. (2016). Fast response dry-type artificial molecular muscles with [c2]daisy chains. Nat. Chem. 8, 625-632. doi: 10.1038/nchem.2513

Jing, Z., Xu, A., Liang, Y. Q., Zhang, Z., Yu, C., Hong, P., et al. (2019). Biodegradable poly(acrylic acid-co-acrylamide)/poly(vinyl alcohol) double network hydrogels with tunable mechanics and high self-healing performance. Polymers 11:952. doi: 10.3390/polym11060952

Kim, Y. S., Liu, M., Ishida, Y., Ebina, Y., Osada, M., Sasaki, T., et al. (2016). Thermoresponsive actuation enabled by permittivity switching in an electrostatically anisotropic hydrogel. Nat. Mater. 14, 1002-1007. doi: $10.1038 /$ nmat4363

Lai, E., Yue, X., Ning, W., Huang, J., Ling, X., and Lin, H. (2019). Three-dimensional graphene-based composite hydrogel materials for flexible supercapacitor electrodes. Front. Chem. 7:660. doi: 10.3389/fchem.2019.00660

Li, W., An, H., Tan, Y., Lu, C., Liu, C., Li, P., et al. (2012). Hydrophobically associated hydrogels based on acrylamide and anionic surface active monomer with high mechanical strength. Soft Matter 8, 5078-5086. doi: 10.1039/c2sm07200a

Li, Z., Su, S., Yu, L., Zheng, Z., and Wang, X. (2018). Preparation of a photo- and thermo-responsive topological gel from anthracene-modified polyrotaxanes. Soft Matter 14, 2767-2771. doi: 10.1039/C8SM00376A

Liang, Z., Liu, C., Li, L., Xu, P., Luo, G., Ding, M., et al. (2016). Double-network hydrogel with tunable mechanical performance and biocompatibility for the fabrication of stem cells-encapsulated fibers and 3D assemble. Sci. Rep. 6:33462. doi: 10.1038/srep33462

Liu, J., Tan, C. S. Y., Yu, Z., Lan, Y., Abell, C., and Scherman, O. A. (2017). Biomimetic supramolecular polymer networks exhibiting both toughness and self-recovery. Adv. Mater. 29:1604951. doi: 10.1002/adma.2016 04951

Liu, Y., Meng, H., Qian, Z., Fan, N., Choi, W., Zhao, F., et al. (2017). A moldable nanocomposite hydrogel composed of a mussel-inspired polymer and a nanosilicate as a fit-to-shape tissue sealant. Angew. Chem. Int. Ed. 56, 4224-4228. doi: 10.1002/anie.201700628

Lou, D., Wang, C., He, Z., Sun, X., Luo, J., and Li, J. (2019). Robust organohydrogel with flexibility and conductivity across the freezing and boiling temperatures of water. Chem. Commun. 55, 8422-8425. doi: 10.1039/C9CC 04239C

Mihajlovic, M., Staropoli, M., Appavou, M. S., Wyss, H. M., PyckhoutHintzen, W., and Sijbesma, R. P. (2017). Tough supramolecular hydrogel based on strong hydrophobic interactions in a multiblock segmented copolymer. Macromolecules 50, 3333-3346. doi: 10.1021/acs.macromol. $7 \mathrm{~b} 00319$
Morelle, X. P., Illeperuma, W. R., Tian, K., Bai, R., Suo, Z., and Vlassak, J. J. (2018). Highly stretchable and tough hydrogels below water freezing temperature. $A d v$. Mater. 30:1801541. doi: 10.1002/adma.201801541

Okumura, Y., and Ito, K. (2001). The polyrotaxane gel: a topological gel by figure-of-eight cross-links. Adv. Mater. 13, 485-487. doi: 10.1002/1521-4095(200104)13:7<485::AID-ADMA485>3.0.CO;2-T

Pan, X., Wang, Q., Ning, D., Dai, L., Liu, K., Ni, Y., et al. (2018). Ultraflexible self-healing guar gum-glycerol hydrogel with injectable, antifreeze, and strain-sensitive properties. ACS Biomater. Sci. Eng. 4, 3397-3404. doi: 10.1021/acsbiomaterials.8b00657

Qin, Z., Niu, R., Tang, C., Xia, J., Ji, F., Dong, D., et al. (2018). A dual-crosslinked strategy to construct physical hydrogels with high strength, toughness, good mechanical recoverability, and shape-memory ability. Macromol. Mater. Eng. 303:1700396. doi: 10.1002/mame.201700396

Qin, Z. H., Dong, D. Y., Yao, M. M., Yu, Q. Y., Sun, X., Guo, Q., et al. (2019). Freezing-tolerant supramolecular organohydrogel with high toughness, thermoplasticity, and healable and adhesive properties. ACS Appl. Mater. Interfaces 11, 21184-21193. doi: 10.1021/acsami.9b05652

Rajan, R., and Matsumura, K. (2018). Development and application of cryoprotectants. Adv. Exp. Med. Biol. 1081, 339-354. doi: 10.1007/978-981-13-1244-1_18

Ricciardi, R., Auriemma, F., Rosa, C. D., and Lauprêtre, F. (2004). X-ray diffraction analysis of poly(vinyl alcohol) hydrogels, obtained by freezing and thawing techniques. Macromolecules 37, 1921-1927. doi: 10.1021/ma $035663 \mathrm{q}$

Rong, Q., Lei, W., Chen, L., Yin, Y., Zhou, J., and Liu, M. (2017). Anti-freezing conductive self-healing organohydrogels with stable strainsensitivity at subzero temperatures. Angew. Chem. Int. Ed. 56, 14159-14163. doi: 10.1002/anie.201708614

Rong, Q., Lei, W., Huang, J., and Liu, M. (2018). Low temperature tolerant organohydrogel electrolytes for flexible solid-state supercapacitors. Adv. Energy Mater. 8:1801967. doi: 10.1002/aenm.201801967

Shi, S., Peng, X., Liu, T., Chen, Y. N., He, C., and Wang, H. (2017). Facile preparation of hydrogen-bonded supramolecular polyvinyl alcohol-glycerol gels with excellent thermoplasticity and mechanical properties. Polymer 111, 168-176. doi: 10.1016/j.polymer.2017.01.051

Wang, M. X., Yang, C. H., Liu, Z. Q., Zhou, J., Xu, F., Suo, Z., et al. (2015). Tough photoluminescent hydrogels doped with lanthanide. Macro. Rapid Commun. 36, 465-471. doi: 10.1002/marc.201400630

Wang, W., Narain, R., and Zeng, H. (2018). Rational design of self-healing tough hydrogels: a mini review. Front. Chem. 6:497. doi: 10.3389/fchem.2018.00497

Wang, Z., Lin, M., Wang, M., Song, X., Zhang, C., Dong, Z., et al. (2018). Polymerizable microsphere-induced high mechanical strength of hydrogel composed of acrylamide. Materials 11:880. doi: 10.3390/ma 11060880

Wegst, U. G., Bai, H., Saiz, E., Tomsia, A. P., and Ritchie, R. O. (2014). Bioinspired structural materials. Nat. Mater. 14, 23-36. doi: 10.1038/nmat4089

Wei, Z., Yang, J. H., Liu, Z. Q., Xu, F., Zhou, J. X., Zrínyi, M., et al. (2015). Novel biocompatible polysaccharide-based self-healing hydrogel. Adv. Funct. Mater. 25, 1352-1359. doi: 10.1002/adfm.201401502

Wei, Z., Yang, J. H., Zhou, J., Xu, F., Zrínyi, M., Dussault, P. H., et al. (2014). Self-healing gels based on constitutional dynamic chemistry and their potential applications. Chem. Soc. Rev. 43, 8114-8131. doi: 10.1039/C4CS 00219A

Wu, J., Wu, Z., Lu, X., Han, S., Yang, B. R., Gui, X., et al. (2019). Ultrastretchable and stable strain sensors based on anti-freezing and self-healing ionic organohydrogels for human motion monitoring. ACS Appl. Mater. Interfaces 11, 9405-9414. doi: 10.1021/acsami.8b20267

Yuk, H., Zhang, T., Lin, S., Parada, G. A., and Zhao, X. (2016). Tough bonding of hydrogels to diverse non-porous surfaces. Nat. Mater. 15, 190-196. doi: $10.1038 /$ nmat4463

Zhang, H., Niu, W., and Zhang, S. (2018). Extremely stretchable, stable and durable strain sensors based on double-network organogels. ACS Appl. Mater. Interfaces 10, 32640-32648. doi: 10.1021/acsami.8b08873

Zhang, Y., Li, Y., and Liu, W. (2015). Dipole-dipole and H-bonding interactions significantly enhance the multifaceted mechanical properties of thermoresponsive shape memory hydrogels. Adv. Funct. Mater. 25, 471-480. doi: 10.1002/adfm.201401989 
Zhang, Y. S., and Khademhosseini, A. (2017). Advances in engineering hydrogels. Science 356:eaaf3627. doi: 10.1126/science. aaf3627

Zhao, X. Q., Wang, M. X., Chen, Y. M., Chen, Z. G., Suo, T., Qian, W., et al. (2019). Puncture-resistant hydrogel: placing molecular complexes along phase boundaries. ACS Appl. Mater. Interfaces 11, 19421-19428. doi: $10.1021 /$ acsami.9b02328

Zhu, P., Deng, Y., and Wang, C. (2017). Graphene/cyclodextrin-based nanocomposite hydrogel with enhanced strength and thermo-responsive ability. Carbohyd. Polym. 174, 804-811. doi: 10.1016/j.carbpol.2017. 06.081
Conflict of Interest: The authors declare that the research was conducted in the absence of any commercial or financial relationships that could be construed as a potential conflict of interest.

Copyright (c) 2020 Zhang, Dong, Guan, Zhang, Chen, Yang, Zhang, Khan, Arfat and Aziz. This is an open-access article distributed under the terms of the Creative Commons Attribution License (CC BY). The use, distribution or reproduction in other forums is permitted, provided the original author(s) and the copyright owner(s) are credited and that the original publication in this journal is cited, in accordance with accepted academic practice. No use, distribution or reproduction is permitted which does not comply with these terms. 